\title{
Execução de Emendas Parlamentares no Distrito Federal: um estudo de caso do parlamento do Distrito Federal
}

\author{
Bruno Ferreira da Paixão ${ }^{1}$
}

\begin{abstract}
Resumo
O presente artigo busca avaliar a aplicação das emendas parlamentares realizadas por deputados distritais no Distrito Federal. Por vezes, as emendas parlamentares são consideradas como ponto de barganha entre o chefe do executivo e a atuação de parlamentares distritais. Este documento busca evidenciar como é realizada a aplicação e execução das emendas propostas por parlamentares. Este artigo abordará temas como o nível de execução orçamentária das unidades orçamentárias e órgãos do Distrito Federal. Além disso demonstrará como os parlamentares do Distrito Federal realizam a alocação dos recursos, quais funções mais recebem emendas e nível de execução das emendas parlamentares. Dessa forma busca-se avaliar a efetiva execução do recurso destinado e efetivamente executado.
\end{abstract}

Palavras-chaves: Emendas parlamentares. Aplicação. Execução.

\section{INTRODUÇÃO}

Segundo definição da Constituição Federal, emenda ao orçamento é uma atividade definida na Constituição Federal de 1988 e recepcionada na Lei Orgânica do Distrito Federal de 8 de junho de 1993. Por definição da Lei Orgânica do Distrito Federal, são destinados $2 \%$ da receita corrente líquida dividida pelos 24 parlamentares como fonte para a apresentação das emendas ao orçamento. "A prerrogativa de apresentar emendas é uma oportunidade dada ao parlamentar para participar da alocação de recursos orçamentários, expressando suas prioridades quanto às políticas públicas [...]”. (FIGUEIREDO; LIMONGI, 2008, p.101).

Cada parlamentar utiliza esse recurso para atender demandas de segmentos e base regional ou que lhe dá sustentação. De autoria dos Deputados Distritais a emenda ao orçamento tem a finalidade de reservar recursos orçamentários para determinada despesa

\footnotetext{
${ }^{1}$ Bruno Ferreira da Paixão.
} 
governamental. Cada parlamentar aloca parte do orçamento que tem direito para financiar uma atividade ou política pública vinculada ao orçamento público.

Este documento busca evidenciar qual o índice de execução orçamentária das emendas parlamentares entre legislaturas e parlamentares. Além disso, demonstra quais órgãos têm um melhor índice de execução e quais temas possuem um maior volume de destinação e execução. Além disso, o trabalho visa verificar se há indícios de discriminação pelo Executivo na autorização de empenhos de emendas individuais de Deputados que não pertencem à base de apoio do governo.

Este trabalho está ancorado na execução orçamentária de emendas parlamentares entre os anos de 2016 e 2019. Ressalta-se que apenas a partir de 2016 o Governo do Distrito Federal passou a divulgar a execução orçamentária das emendas parlamentares. O estudo tem o objetivo evidenciar o comportamento da execução orçamentária das emendas destinadas por parlamentares ao orçamento geral do Distrito Federal. A análise de dados leva em consideração 0 percentual de recurso empenhado em atendimento a emendas nos exercícios de 2016, 2017, 2018 e 2019.

\section{METODOLOGIA}

Os dados apresentados foram coletados do portal da transparência do Distrito Federal, sítio oficial que consolida dados os oficiais do governo. O portal organiza e concentrada os dados provenientes do Sistema de Gestão Governamental - SIGGO, ferramenta que realiza a gestão financeira e orçamentária das contas públicas do Distrito Federal.

A base de dados foi coletada entre os anos de 2016 e 2019. Cumpre ressaltar que apenas a partir de 2016 os dados de emendas parlamentares foram disponibilizados de forma estruturada e acessível.

Para analisar e consolidar os dados, utilizou-se o pacote de soluções Power BI para visualizações de dados. De forma adicional foi utilizada a linguagem de programação Python com as bibliotecas pandas para análise de dados e sklearn para técnicas de limpeza de dados e aprendizado de máquina.

Pandas: biblioteca de software escrita para a linguagem de programação Python para manipulação e análise de dados.

Sklearn: biblioteca de aprendizado de máquina de código aberto para a linguagem de programação Python. 
Os dados foram coletados do portal da transparência do Distrito Federal e consolidados em uma tabela discriminando os atributos para análise. Os dados são atualizados diariamente tendo como fonte de informação o Sistema de Gestão Integrada do Distrito Federal - SIGGO, ferramenta responsável por toda gestão orçamentária e financeira do Governo do Distrito Federal.

Tabela 1 - Estrutura de Dados

\begin{tabular}{l|l}
\hline \multicolumn{1}{c|}{ Campo } & \multicolumn{1}{c}{ Descrição } \\
\hline Ano & Ano de execução da emenda. \\
\hline Despesa Autorizada & $\begin{array}{l}\text { Crédito inserido na lei do orçamento para atender uma despesa } \\
\text { programada. }\end{array}$ \\
\hline Empenho & $\begin{array}{l}\text { Compromisso que o estado cria com um credor prevendo um futuro } \\
\text { pagamento desde que as obrigações assumidas por ele sejam cumpridas. }\end{array}$ \\
\hline Liquidado & $\begin{array}{l}\text { Verificação de que um credor cumpriu com sua obrigação e possui o } \\
\text { direito de receber aquele crédito. }\end{array}$ \\
\hline Pago & Valor pago de despesas do exercício corrente. \\
\hline Categoria Econômica & $\begin{array}{l}\text { Classificação das receitas e despesas para uma avaliação do efeito } \\
\text { econômico. }\end{array}$ \\
\hline Fonte de Recurso & Origem do Recurso. \\
\hline Função & Área de atuação da Administração Pública. \\
\hline Elemento de Despesa & Indica os objetos de gastos. \\
\hline Grupo de Natureza da & $\begin{array}{l}\text { Agrega elementos de despesa com as mesmas características quanto ao } \\
\text { objeto do gasto. }\end{array}$ \\
\hline $\begin{array}{l}\text { Despesa } \\
\text { Aplicação }\end{array}$ & $\begin{array}{l}\text { Indica se os recursos serão aplicados diretamente pelo órgão ou sob a } \\
\text { forma de transferência a outras entidades públicas ou privadas. }\end{array}$ \\
\hline Programa de & $\begin{array}{l}\text { Conjunto organizado das ações que serão executadas de acordo com as } \\
\text { possibilidades financeiras do governo. }\end{array}$ \\
\hline Trabalho & Região Administrativa. \\
\hline RA & Identifica a natureza básica das ações de uma determinada área de atuação. \\
\hline Subfunção & Descrição detalhada com a regionalização das ações e projetos do governo. \\
\hline Subtítulo & Órgãos e entidades encarregadas de gerir os recursos públicos. \\
\hline Unidade Gestora & Nome do parlamentar. \\
\hline Nome do Autor & Razão entre despesa autorizada e empenho \\
\hline Execução & Fonte: Portal da Transparência do Distrito Federal - 2019. \\
\hline &
\end{tabular}

\section{RESULTADOS}

O volume de recursos destinado anualmente para execução de emendas parlamentares corresponde a $2 \%$ da receita corrente líquida anual do Distrito Federal. O valor total é dividido igualmente entre os 24 parlamentares. Como o valor da receita corrente líquida é variável, o valor destinado à execução de emendas parlamentares varia conforme a Lei Orçamentária Anual. Entre os anos de 2016 e 2019 houve uma despesa autorizada de cerca de $\mathrm{R} \$ 1,7$ bilhão para custeio de emendas parlamentares. $\mathrm{O}$ ano de 2018 foi o período com maior recurso liberado para custear emendas parlamentares (ver Gráfico 1). (Bruno Paixão, 2019). 
Gráfico 1 - Despesa autorizada para custeio de emendas parlamentares no Distrito Federal

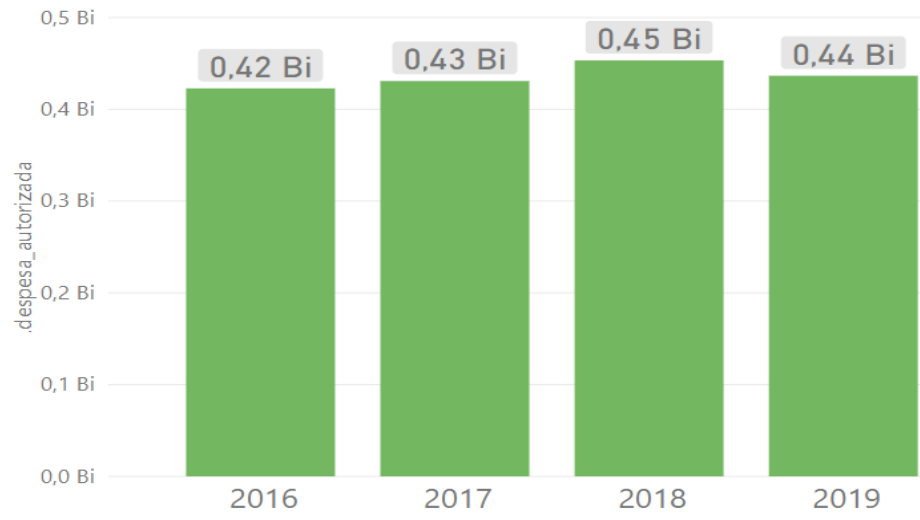

Fonte: Portal da Transparência do Distrito Federal - 2019.

Entre os anos de 2016 e 2018, os parlamentares que compunham esta legislatura destinaram os recursos para a financiar ações com categoria econômica classificadas como investimento. Nos referidos anos, mais de $60 \%$ do percentual da despesa autorizada de emendas parlamentares foi destinado a ações que financiassem novas ações de investimento. (DISTRITO FEDERAL, 2019) Em 2019, com uma nova legislatura na Câmara Legislativa do Distrito Federal, esse percentual de aplicação em investimento caiu para cerca de $52 \%$, o percentual restante corresponde ao financiamento de despesas correntes do estado, indicando no primeiro ano uma mudança no perfil das emendas destinadas (ver Gráfico 2) (DISTRITO FEDERAL, 2019).

Gráfico 2 - Percentual de Despesa Autorizada aplicado por categoria Econômica

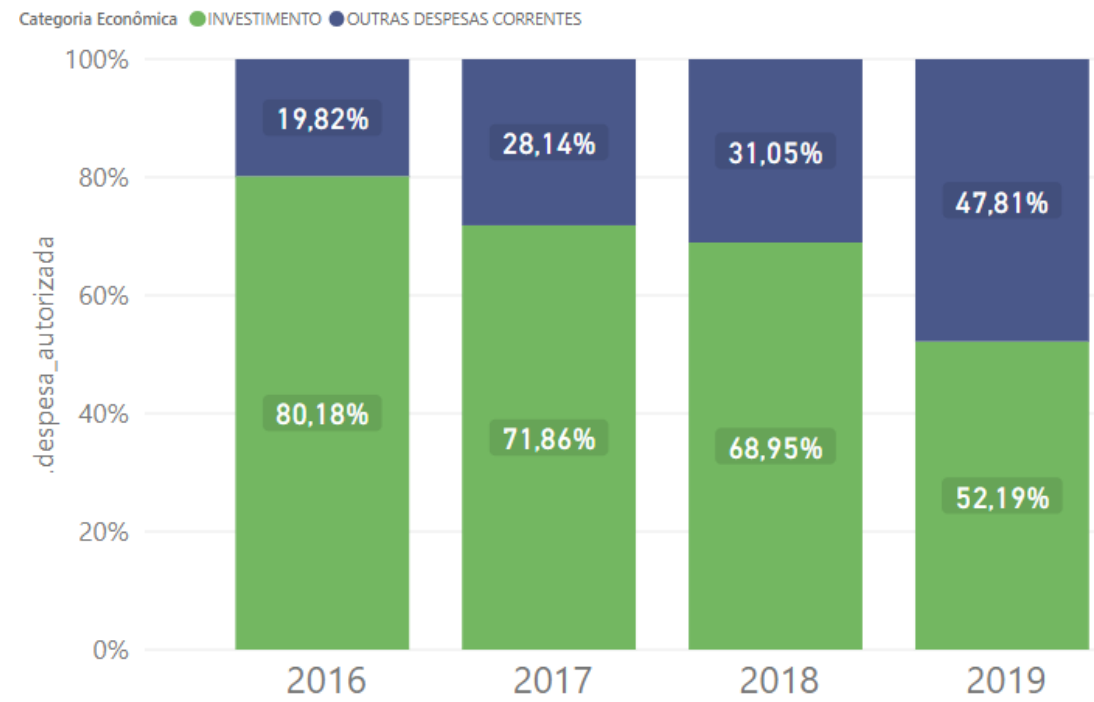

Fonte: Portal da Transparência do Distrito Federal - 2019. 
Ao analisar as funções econômicas de financiamento dos recursos provenientes de emendas, observamos que os parlamentares alocam suas escolhas prioritariamente em ações que executem atividades voltadas para o urbanismo da cidade. Entre 2016 e 2019, a função orçamentária classificada como urbanismo recebeu cerca de $40 \%$ das indicações para recebimento dos recursos alocados por parlamentares (ver Tabela 2). Grande parte das ações agrupadas na função de urbanismo são destinadas a obras de pequeno valor executadas nas Regiões Administrativas.

Tabela 2 - Despesa e Percentual autorizado por Função e Ano

\begin{tabular}{|c|c|c|c|c|c|c|c|c|c|c|}
\hline Ano & 2016 & & 2017 & & 2018 & & 2019 & & Total & \\
\hline Função & Despesa & $\%$ & Despesa & $\%$ & Despesa & $\%$ & Despesa & $\%$ & Despesa & $\%$ \\
\hline URBANSMO & R\$221.985.000 & $52.50 \%$ & RS172.006.606 & $39,93 \%$ & R\$163.625.492 & $36,10 \%$ & R\$142.732.467 & $32.69 \%$ & RS700.349.565 & $40,17 \%$ \\
\hline EDUCACAO & $R S 48.790 .000$ & $11,54 \%$ & RS73.350.855 & $17,03 \%$ & R\$101.348.848 & $22,36 \%$ & R\$95.713.844 & $21,92 \%$ & $\mathrm{R} \$ 319.203 .547$ & $18,31 \%$ \\
\hline SAUDE & R\$23.835.000 & $5,64 \%$ & $R \$ 42.018 .001$ & $9,75 \%$ & $R \$ 36.432 .460$ & $8,04 \%$ & R\$35.314.882 & $8,09 \%$ & R\$137.600.343 & $7,89 \%$ \\
\hline TRANSPORTE & RS16.901.580 & $4,00 \%$ & $R \$ 32.660 .000$ & $7,58 \%$ & RS30.106.000 & $6,64 \%$ & R\$32.541.941 & $7,45 \%$ & RS112.209.521 & $6,44 \%$ \\
\hline DESPORTO E LAZER & $R \$ 26.301 .420$ & $6,22 \%$ & $R \$ 23.743 .000$ & $5,51 \%$ & RS27.200.712 & $6,00 \%$ & R\$27.011.941 & $6,19 \%$ & RS104.257.073 & $5,98 \%$ \\
\hline CULTURA & RS26.320.000 & $6,22 \%$ & RS18.343.000 & $4.26 \%$ & $R \$ 20.742 .856$ & $4,58 \%$ & $R \$ 32.449 .999$ & $7,43 \%$ & R\$97.855.855 & $5,61 \%$ \\
\hline DIREITOS DA CIDAOANA & R\$12.187.000 & $2.88 \%$ & RS23.370.916 & $5,42 \%$ & $R \$ 20.452 .108$ & $4,51 \%$ & $R \$ 27.960 .000$ & $6.40 \%$ & R\$83.970.024 & $4,82 \%$ \\
\hline SEGURANCA PUBUCA & RS4.750.000 & $1,12 \%$ & RS6.323.572 & $1,47 \%$ & R\$13.140.000 & $2,90 \%$ & R\$14.729.000 & $3,37 \%$ & R\$38.942.572 & $2,23 \%$ \\
\hline ENERGIA & $R \$ 2.500 .000$ & $0,59 \%$ & $R \$ 6.250 .000$ & $1,45 \%$ & $R \$ 10.266 .000$ & $2,26 \%$ & R\$5.971.941 & $1,37 \%$ & $\mathrm{R} \$ 24.987 .941$ & $1,43 \%$ \\
\hline COMERCDO E SERULÇOS & $R \$ 6.650 .000$ & $1,57 \%$ & $R \$ 3.428 .544$ & $0.80 \%$ & $R S 4.480 .000$ & $0,99 \%$ & $R \$ 9.300 .000$ & $2.13 \%$ & R\$23.858.544 & $1,37 \%$ \\
\hline AGAJCULTURA & RS5.125.000 & $1,21 \%$ & $R \$ 5.760 .000$ & $1,34 \%$ & $R \$ 7.040 .000$ & $1,55 \%$ & R\$4.190.000 & $0,96 \%$ & $\mathrm{R} \$ 22.115 .000$ & $1,27 \%$ \\
\hline ADMINSTRACZOO & RS7.200.000 & $1,70 \%$ & $R \$ 6.980 .000$ & $1,62 \%$ & $R \$ 3.945 .000$ & $0,87 \%$ & R\$3.120.000 & $0,71 \%$ & R\$21.245.000 & $1,22 \%$ \\
\hline ENCARGOS ESPECALS & & & R57.000.000 & $1,62 \%$ & $R \$ 7.000 .000$ & $1,54 \%$ & & & R\$14.000.000 & $0,80 \%$ \\
\hline GESTAO AMBIENTAL & $R \$ 5.600 .000$ & $1,32 \%$ & $R \$ 2.173 .572$ & $0,50 \%$ & $R \$ 3.047 .856$ & $0,67 \%$ & R\$935.000 & $0,21 \%$ & R\$11.756.428 & $0,67 \%$ \\
\hline LEGSLATIVA & RS6.890.000 & $1,63 \%$ & & & $R \$ 2.218 .856$ & $0,49 \%$ & & & RS9.108.856 & $0,52 \%$ \\
\hline TRABALHO & RS1.150.000 & $0,27 \%$ & R\$5.350.000 & $1,24 \%$ & $R S 400.000$ & $0,09 \%$ & $R \$ 800.000$ & $0,18 \%$ & R57.700.000 & $0,44 \%$ \\
\hline ASSISTENECIA SOCIAL & RS4. 180.000 & $0,99 \%$ & RS899.000 & $0,21 \%$ & $R \$ 962.000$ & $0,21 \%$ & $R \$ 700.000$ & $0,16 \%$ & RS6.741.000 & $0,39 \%$ \\
\hline EESENCIAL A A JuSTIÇA & $R \$ 450.000$ & $0,11 \%$ & RS $\$ 800.000$ & $0,19 \%$ & $R \$ 900.000$ & $0,20 \%$ & RS1.500.000 & $0,34 \%$ & RS3.650.000 & $0,21 \%$ \\
\hline SANEAMENTO & $R \$ 2.000 .000$ & $0,47 \%$ & RSO & $0,00 \%$ & RSO & $0,00 \%$ & R\$1.100.000 & $0,25 \%$ & R\$3.100.000 & $0,18 \%$ \\
\hline NOCIAAEAA & & & $R \$ 350.000$ & $0,08 \%$ & & & & & RS350.000 & $0,02 \%$ \\
\hline CEENCLA E TECNOLOGIA & & & & & $R S O$ & $0,00 \%$ & $R \$ 300.000$ & $0,07 \%$ & RS300.000 & $0,02 \%$ \\
\hline HABTTCCAO & & & & & & & $R \$ 200.000$ & $0,05 \%$ & RS200.000 & $0,01 \%$ \\
\hline Total & $\mathrm{R} \$ 422.815 .000$ & $100,00 \%$ & $\mathrm{R} \$ 430.807 .066$ & $100,00 \%$ & R\$453.308.188 & $100,00 \%$ & RS436.571.015 & $100,00 \%$ & RS1.743.501.269 & $100,00 \%$ \\
\hline
\end{tabular}

Fonte: Portal da Transparência do Distrito Federal - 2019.

Com a sanção da Lei do Programa de Descentralização Administrativa e Financeira (PDAF), em 2017, a função educação começou a receber um volume expressivo de recursos provenientes de emendas parlamentares. Cumpre destacar que o Programa de Descentralização Administrativa e Financeira (PDAF) foi: “[...] criado para gerar autonomia financeira nas unidades escolares e coordenações regionais de ensino (CREs), nos termos do projeto político-pedagógico e planos de trabalho de cada uma." (DISTRITO FEDERAL, 2019). Assim diversos parlamentares passaram a indicar recursos para esta função, chegando ao volume de $15 \%$ do total destinado em 2019. Entre 2016 e 2019, o Programa de Descentralização Administrativa e Financeira (PDAF) recebeu cerca de R\$150 milhões em emendas parlamentares (ver Gráfico 3) (Bruno Paixão, 2019). 
Gráfico 3 - Despesa Autorizada para o Programa de Descentralização Administrativa e Financeira (PDAF) por ano

PDAF • PDAF DESTINADO • PDAF NÃO DESTINADO

2016

2017

$2018 \quad 14,01 \%$

2019

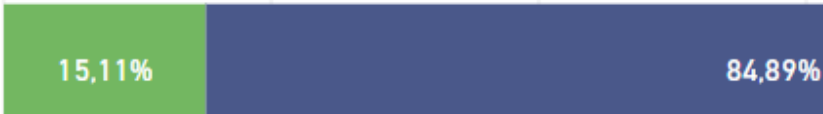

$\begin{array}{llllll}0 \% & 20 \% & 40 \% & 60 \% & 80 \% & 100 \%\end{array}$

Fonte: Portal da Transparência do Distrito Federal - 2019.

Um importante aspecto de análise é avaliar o percentual de recursos destinados das emendas parlamentares aos órgãos executores dos recursos. Historicamente a Companhia Urbanizadora da Nova Capital - Novacap, empresa responsável por executar as principais obras do Distrito Federal, recebe a maior destinação das emendas parlamentares, cerca de 22\% entre 2016 e 2019 (Bruno Paixão, 2019). Todavia, com a institucionalização do Programa de Descentralização Administrativa e Financeira (PDAF) da Secretaria de Educação, este órgão passou a receber um expressivo volume de recursos, com incremento de 6,5\% em orçamento alocado quando comparado os exercícios de 2016 a 2019 (Bruno Paixão, 2019), conforme a Tabela 3 abaixo. 
Tabela 3 - Despesa e Percentual autorizado por Órgão e Ano

\begin{tabular}{|c|c|c|c|c|c|c|c|c|c|c|}
\hline hidade Gestora & Despesa & $\%$ & Despesa & $\%$ & Despesa & $\%$ & Despesa & $\%$ & Despesa & $\%$ \\
\hline $\begin{array}{l}\text { MPANHA URB DA NOVA CAPITAL DO BRASL- } \\
\text { WACAP }\end{array}$ & $R \$ 73.771,000$ & $17,45 \%$ & R598.478.918 & $22,86 \%$ & RS113.114.636 & $24,95 \%$ & R\$112,983.586 & $25,88 \%$ & $\mathrm{R} \$ 398.348 .140$ & $22,85 \%$ \\
\hline SECRETARIA DE ESTADD DE EDUCACGAO DO DF & $R 558.421,420$ & $13,82 \%$ & $R S 67.880 .855$ & $15,76 \%$ & 596.198 .848 & $21,22 \%$ & $R \$ 88.313 .844$ & $20,23 \%$ & $R \$ 310.814 .967$ & $17,83 \%$ \\
\hline FUNDO DE SALOE DO DISTRITO FEDERAL & $R \$ 23.825 .000$ & $5,63 \%$ & RS41.518,001 & $9,64 \%$ & 32.460 & $7,57 \%$ & .814 .882 & $7,75 \%$ & $\mathrm{R} \$ 133.490 .343$ & $7,66 \%$ \\
\hline DEPTO DE ESTRNDAS E ROOAGEM DO DF - DCR/DF & $R \$ 6.930 .000$ & $1,64 \%$ & $R \$ 30,300,000$ & $7,03 \%$ & R\$25.050.000 & $5,53 \%$ & $R \$ 16.761 .940$ & $3,84 \%$ & $\mathrm{R} \$ 79.041 .940$ & $4,53 \%$ \\
\hline SECRETARIA DE ESTADO DE OQRAS E INFRAEST. DO DF & $R \$ 45.630,000$ & $10,79 \%$ & $R \$ 6.417,144$ & $1,49 \%$ & $R \$ 6.516 .000$ & $1,44 \%$ & RS8.601.941 & $1,97 \%$ & $R \$ 67.165 .085$ & $3,85 \%$ \\
\hline ADMINISTRACGAO REG ONAL DE CELANNDAA & $R \$ 21.190,000$ & $5,01 \%$ & $R \$ 21.802,144$ & $5,06 \%$ & R\$17.477.856 & $3,86 \%$ & $\operatorname{R} \$ 2.741 .940$ & $0,63 \%$ & $\mathrm{R} \$ 63.211 .940$ & $3,63 \%$ \\
\hline SEC DE POLITCAS P/CRIANCAS, ADOLESC. E JUVENTUDE & $R \$ 7.920 .000$ & $1,87 \%$ & $R \$ 14,147,144$ & $3,28 \%$ & $R 516.504,108$ & $3,64 \%$ & $R \$ 22,960.000$ & $5,26 \%$ & $\mathrm{R} \$ 61.531 .252$ & $3,53 \%$ \\
\hline SECRETARIA DE ESTADO DE ESPORTE E LAZZR DO DF & RSO & $0,00 \%$ & R\$11.959.772 & $2,78 \%$ & R\$11.277.856 & $2,49 \%$ & 000 & $6,89 \%$ & $\mathrm{R} \$ 53.337 .628$ & $3,06 \%$ \\
\hline SECRETARIA DE EST. DE CUTTURA DO DISTRITO FEDERAL & $R \$ 17.970 .000$ & $4,25 \%$ & RS15. & $3,70 \%$ & 2.856 & $3,33 \%$ & & & $\mathrm{R} \$ 49.012 .856$ & $2,81 \%$ \\
\hline ADMINISTRACTOO REG ONAL DE TAGUATINGA & $R \$ 15.950,000$ & $3,77 \%$ & 0.000 & $2,95 \%$ & & $1,08 \%$ & & $0,35 \%$ & 80.000 & $2,01 \%$ \\
\hline ACMINISTRACZTOO REGOONAL DE AGGUAS CLARAS & $R \$ 11.750 .000$ & $2,78 \%$ & $R \$ 13,640,000$ & $3,17 \%$ & $R \$ A, 900,000$ & $1,08 \%$ & 1.000 & $0,93 \%$ & $\mathrm{R} \$ 34.350 .000$ & $1,97 \%$ \\
\hline ADMINISTRACTKO REG ONAL DE BRAZZANDIA & R\$11.154,000 & $2,64 \%$ & $R \$ 5,800,572$ & $1,35 \%$ & $R \$ 6.265 .000$ & $1,38 \%$ & & $1,35 \%$ & $\mathrm{R} \$ 29.1$ & $1,67 \%$ \\
\hline SECRETARIA DE EST. DE CUUTURA E ECON CRIAT. DO DF & & & & & & & $R \$ 28.820 .000$ & $6,60 \%$ & $\mathrm{R} \$ 28.820 .000$ & $1,65 \%$ \\
\hline ADMINISTRAÇBO REGGONAL DE FLANQLTINA & 0.000 & $2,13 \%$ & 000 & $1,68 \%$ & & $1,46 \%$ & & $0,37 \%$ & $\mathrm{R} \$ 24.450 .000$ & $1,40 \%$ \\
\hline ADMINISTRAÇAO REG OONAL DE SR̃O SEAASTIÄO & $R S 10,400,000$ & $2,46 \%$ & $R \$ 5,102,000$ & $1,18 \%$ & R\$A.150,000 & $0,92 \%$ & $R \$ 2,500,000$ & $0,57 \%$ & $\mathrm{R} \$ 22.152 .000$ & $1,27 \%$ \\
\hline $\begin{array}{l}\text { SECRETARIA DE AGRIC. ABASTECMENTO E DESEN } \\
\text { RURAL }\end{array}$ & $R \$ 4,475,000$ & $1,06 \%$ & $R \$ 7,130,000$ & $1,66 \%$ & $R \$ 5.260 .000$ & $1,16 \%$ & $R \$ 3,990,000$ & $0,91 \%$ & $\mathrm{R} \$ 20.855 .000$ & $1,20 \%$ \\
\hline SECR. TRAB. DES. SOCIAL MULHERES IG. RACINL DH & $R \$ 3.350 .000$ & $0,79 \%$ & $R \$ 6.849,000$ & $1,59 \%$ & $R \$ 4.227,000$ & $0,93 \%$ & 80.000 & $1,32 \%$ & $\mathrm{R} \$ 20.206 .000$ & $1,16 \%$ \\
\hline ADMINISTRACTAO REGONAL DO GAMA & $R \$ 9.169 .000$ & $2,17 \%$ & 000 & $0,64 \%$ & 000 & $0,96 \%$ & & $0,74 \%$ & $\mathrm{R} \$ 19.500 .941$ & $1,12 \%$ \\
\hline ADMINISTRACG̈OOO REGOCNAL DE SAMAMEAAA & $R \$ 5.750 .000$ & $1,36 \%$ & $R \$ 4,200,000$ & $0,97 \%$ & $R \$ 3.750 .000$ & $0,83 \%$ & & $0,94 \%$ & 320.000 & $1,02 \%$ \\
\hline ADMINISTRACGTO REGONAL DO RECANTO DAS EMAS & $R \$ 5.530 .000$ & $1,31 \%$ & $R \$ 1.900,000$ & $0,44 \%$ & 1.856 & $0,99 \%$ & 0.000 & $1,10 \%$ & $\mathrm{R} \$ 16.717 .856$ & $0,96 \%$ \\
\hline COMPANHIA DO METROPOUTANO DO OF - METRO-DF & $R \$ 3.152 .580$ & $0,75 \%$ & $R \$ 0$ & $0,00 \%$ & $R \$ 2.580,000$ & $0,57 \%$ & $R \$ 10,641.941$ & $2,44 \%$ & $\mathrm{R} \$ 16.374 .521$ & $0,94 \%$ \\
\hline SECRETARIA DE PLANELAMENTO, ORCAMENTO E GESTIOO & & & $R \$ 7,000,000$ & $1,62 \%$ & $R \$ 8.500 .000$ & $1,88 \%$ & $R \$ 850.000$ & $0,19 \%$ & $\mathrm{R} \$ 16.350 .000$ & $0,94 \%$ \\
\hline POLIOA CIVIL DO DSTRRTO FEDERAL - PCDF & $R \$ 100,000$ & $0,02 \%$ & $R \$ 1.923 .572$ & $0,45 \%$ & $R \$ 5.900 .000$ & $1,30 \%$ & RS6.891.000 & $1,58 \%$ & $\mathrm{R} \$ 14.814 .572$ & $0,85 \%$ \\
\hline ADMINISTRACATIO REGONAL DE SANTA MARIA & $R \$ 6.500,000$ & $1,54 \%$ & $R \$ 4,400,000$ & $1,02 \%$ & $R \$ 2.270 .000$ & $0,50 \%$ & $R \$ 1,000,000$ & $0,23 \%$ & $\mathrm{R} \$ 14.170 .000$ & $0,81 \%$ \\
\hline SECRETARIA DE EST. DE JUSTTCA E GDADANIA DO DF & $R \$ 5.265 .000$ & $1,25 \%$ & $R \$ 6.823 .772$ & 1,5 & & & & $0,23 \%$ & $\mathrm{R} \$ 13.788 .772$ & $0,79 \%$ \\
\hline ADMINISTRACATO REGCONAL DO PARANOÁ & $R \$ 6.650 .000$ & $1,57 \%$ & $R \$ 950,000$ & $0,22 \%$ & $R \$ 2.610,000$ & $0,58 \%$ & $R \$ 500.000$ & $0,11 \%$ & $\mathrm{R} \$ 10.710 .000$ & $0,61 \%$ \\
\hline $\begin{array}{l}\text { SECRETARIA DE EST.DE TRANSPORTE E MOBIUDADE DO } \\
\text { DF }\end{array}$ & $R \$ 2.479,000$ & $0=5$ & & & $R \$ 5.500,000$ & $1,21 \%$ & RS1.500,000 & $0,34 \%$ & $\mathrm{R} \$ 9.479 .000$ & $0,54 \%$ \\
\hline 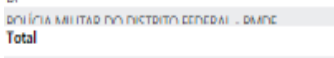 & $\mathrm{R} \$ 422.815 .000$ & $100,00 \%$ & $\$ 430.807 .066$ & $100,00 \%$ & $\begin{array}{l}n+1=4 \cap n m \\
\$ 453.308 .188\end{array}$ & $100,00 \%$ & $\begin{array}{l}\text { neinen } \\
2 \$ 436.571 .015\end{array}$ & 100,00 & 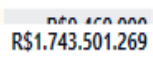 & $100 \hat{0}$ \\
\hline
\end{tabular}

Fonte: Portal da Transparência do Distrito Federal - 2019.

Vencida a etapa de alocação dos recursos provenientes de emendas parlamentares, temos um passo seguinte que é a efetiva execução e materialização da destinação do recurso. A etapa de execução é considerada uma fase crucial, pois ela concretiza a intenção do parlamentar que foi iniciada com a alocação e autorização da despesa. A execução da emenda parlamentar é realizada por conjunto de fatores. O primeiro é a vontade política do Poder Executivo para liberação do recurso. Outro fator que impacta na execução da emenda é existência de projetos e atos preparatórios para a ação que receberá o recurso.

Assim uma emenda é executada quando:

Quando uma emenda é criada, as unidades executoras (Secretarias, Administrações Regionais) ficam aguardando que o parlamentar autorize sua utilização. Para tanto, o deputado encaminha uma solicitação por meio do SISCONEP à Casa Civil que, por sua vez, analisa e informa à Secretaria de Estado de Planejamento, Orçamento e Gestão - SEPLAG sobre o pedido do parlamentar. Na SEPLAG, é feita a liberação do 
orçamento. As unidades executoras são sinalizadas por meio do SISCONEP que o orçamento já está disponível para utilização.

Assim temos a fase de empenho que segundo o SISCONEP (2019) é:

O valor empenhado é o reservado para honrar compromisso com determinada despesa firmada. $\mathrm{O}$ valor liquidado é referente a despesas que já foram recebidas, com nota fiscal, em processo de pagamento.[...] NA PRÁTICA: a unidade executora da pavimentação da rua, que recebeu o orçamento de emenda parlamentar no valor de $\mathrm{R} \$ 50.000$ para essa finalidade, recebeu por meio do SISCONEP a informação de que está liberado o início da obra. Decide, então, escolher uma empresa para realizá-la. A empresa cobrará $\mathrm{R} \$ 50.000$ por esse serviço. Terminados todos os trâmites de contratação, é necessário EMPENHAR esse valor, ou seja, reservar esse orçamento para que, quando o trabalho for entregue, tenha o recurso reservado para pagar. Terminada a obra e entregue a nota fiscal, é hora de LIQUIDAR a despesa, ou seja, informar que o que foi contratado foi cumprido e dar início aos trâmites do pagamento.

Dessa forma, é necessário avaliar o "índice de execução orçamentária” que é dado pela razão entre o "valor empenhado" no período em função da "despesa autorizada". Este índice é fundamental para avaliar a capacidade de execução da emenda destinada, do órgão que recebe o recurso e das funções que agrupam as ações financiadas com a emenda.

Quando uma despesa autorizada passa para fase de empenho, ela cria um compromisso do estado com terceiros em virtude de uma prestação serviço. Segundo definição do Manual de Orçamento do Distrito Federal (2019) o empenho é definido como: "Compromisso que o estado cria com um credor prevendo um futuro pagamento desde que as obrigações assumidas por ele sejam cumpridas.”.

No período avaliado, entre os anos de 2016 e 2019, é possível observar (ver Gráfico 4) que foram empenhados cerca de R \$ 579 milhões. Contudo, verifica-se que o valor empenhado é bem inferior ao valor alocado em despesa autorizada. 
Gráfico 4 - Despesa autorizada e Despesa Empenhada por Ano

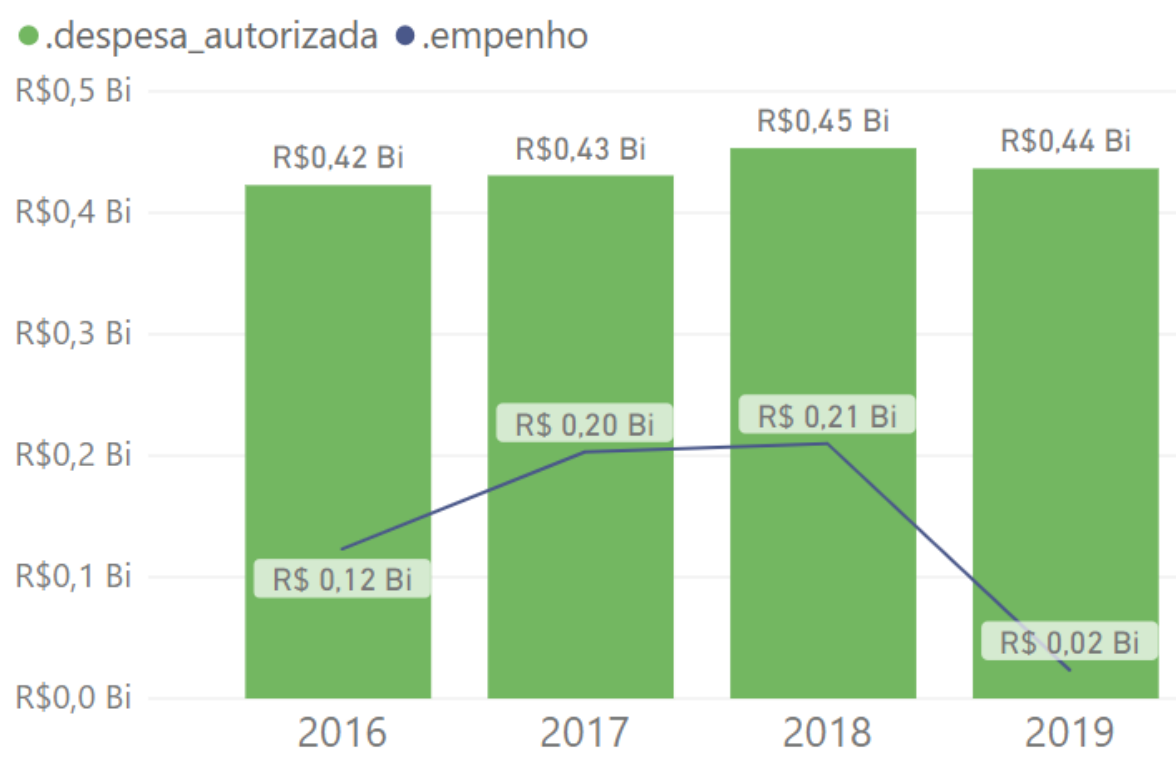

Fonte: Portal da Transparência do Distrito Federal - 2019.

O índice de execução orçamentária é dado pela razão entre o valor empenhado, ou compromisso firmado pelo estado em relação à despesa autorizada, e a interação de alocação de recursos. Nesse contexto, verifica-se que desde 2016 jamais se empenhou mais do que 50\% da despesa inicialmente autorizada (ver Gráfico 5).

Gráfico 5 - Índice de Empenho por Ano

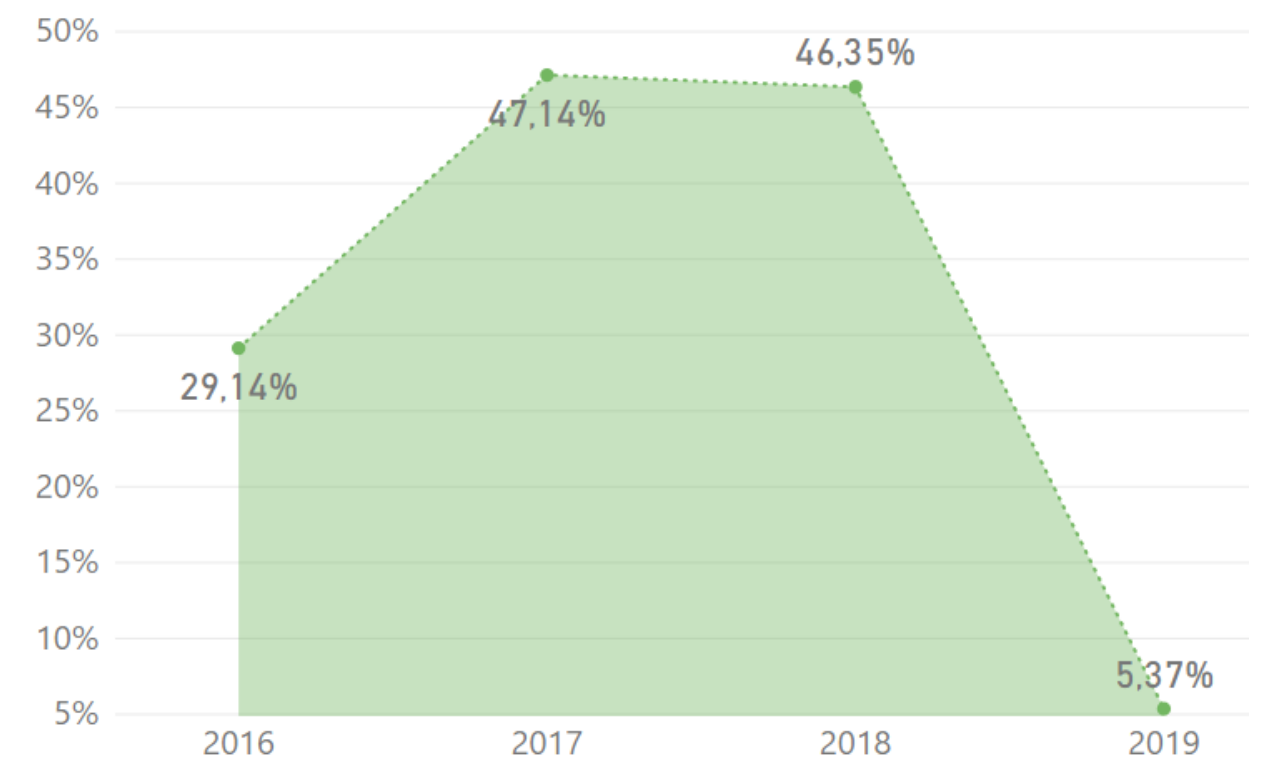

Fonte: Portal da Transparência do Distrito Federal - 2019.

Ao analisar o índice de execução de empenho por função (ver Gráfico 6), é possível observar um movimento inverso, nem sempre as funções com maior volume de recursos destinado são a que melhor empenham e executam o valor. A função 
urbanismo recebeu maior volume de recursos em despesa alocada por emendas parlamentares, porém executou menos de 30\% proporcionalmente. Assim é possível classificar o índice de execução em faixas de desempenho, a saber:

Tabela 4 - Escala de Execução Orçamentária

\begin{tabular}{c|l}
\hline Baixo Desempenho & Empenho de até 33\% da despesa autorizada \\
\hline Médio Desempenho & Empenho de até 66\% da despesa autorizada \\
\hline Alto Desempenho & Empenho de até $100 \%$ da despesa autorizada \\
\hline
\end{tabular}

Fonte: Portal da Transparência do Distrito Federal - 2019.

Tabela 5 - Índice de Empenho por Função

\begin{tabular}{|c|c|c|c|}
\hline Inção & mpenho & Despesa Autorizada & Valor Empenhado \\
\hline CULTURA & $62,63 \%$ & $R \$ 97.855 .855$ & $R \$ 61.287 .267$ \\
\hline TRABALHO & $45 \%$ & $\mathrm{R} \$ 7.700 .000$ & $R \$ 3.500 .000$ \\
\hline SAÚdE & $44,85 \%$ & $\mathrm{R} \$ 137.600 .343$ & $R \$ 61.718 .102$ \\
\hline DIREITOS DA CIDA & $41,58 \%$ & $\mathrm{R} \$ 83.970 .024$ & $\mathrm{R} \$ 34$ \\
\hline AGRICULTURA & $35,64 \%$ & $\mathrm{R} \$ 22.115 .000$ & $R \$ 7.881 .120$ \\
\hline DESPORTO E LAZER & $35,19 \%$ & $\mathrm{R} \$ 104.257 .073$ & $R \$ 36.685 .065$ \\
\hline EDUCAÇ̃̃O & & $\mathrm{R} \$ 3$ & R\$ 103.440 .991 \\
\hline URBANISMO & $28,21 \%$ & $\mathrm{R} \$ 70$ & R\$ 197.576.905 \\
\hline GESTÃO AM & & $\$ 28$ & 523 \\
\hline ENERGIA & $12,47 \%$ & $R \$ 24.987 .941$ & 537 \\
\hline ADIMINISTRAÇÃO & $7,57 \%$ & $R \$ 21.245 .000$ & 395 \\
\hline SEGURANÇA PÚBLI & $6,95 \%$ & $R \$ 38.9$ & $R \$ 2.706 .892$ \\
\hline TRANSPORTE & $3,86 \%$ & $\mathrm{R} \$ 112.209 .521$ & $R \$ 4.325 .873$ \\
\hline ASSISTÊNCIA SC & $1,53 \%$ & $R \$ 6.741 .000$ & $R \$ 103.175$ \\
\hline ENCARGOS ESPECIAIS & $0,00 \%$ & $\mathrm{R} \$ 14.000 .000$ & $\mathrm{R} \$ 0$ \\
\hline
\end{tabular}

Fonte: Portal da Transparência do Distrito Federal - 2019.

Quando analisado o índice anualmente, vemos que a função cultura se destaca por conseguir índices superiores aos 50\% de índice de empenho (ver Tabela 5). Isso se deve ao fato de que boa parte das ações são referentes à prestação de serviços e shows, ação com maior facilidade para execução do ponto de vista administrativo. Outras funções da despesa têm uma grande oscilação no índice de execução, isso se deve ao fato de que algumas das ações financiadas são obras de grande porte que dependem de projetos de arquitetura e engenharia e licenças ambientais (ver Tabela 6). 
Tabela 6 - Índice de Empenho por Função e Ano

\begin{tabular}{|c|c|c|c|c|c|}
\hline Função & 16 & 017 & 018 & 019 & Total \\
\hline CULTURA & $50,00 \%$ & $88,64 \%$ & $115,72 \%$ & $24,24 \%$ & $62,63 \%$ \\
\hline TRABALHO & $0,00 \%$ & $56,07 \%$ & $125,00 \%$ & $0,00 \%$ & $45,45 \%$ \\
\hline SAÚDE & $104,88 \%$ & $66,65 \%$ & $23,92 \%$ & $0,00 \%$ & $44,85 \%$ \\
\hline DIREITOS DA CIDAC & $73,41 \%$ & $47,83 \%$ & $72,30 \%$ & $0,00 \%$ & $41,58 \%$ \\
\hline AGRICULTURA & $80,15 \%$ & $19,89 \%$ & $37,33 \%$ & $0,00 \%$ & $35,64 \%$ \\
\hline DESPORTO E L & $19,86 \%$ & $46,28 \%$ & $56,95 \%$ & $18,45 \%$ & $35,19 \%$ \\
\hline EDUCAÇÃO & $48,45 \%$ & $53,02 \%$ & $39,38 \%$ & $1,04 \%$ & $32,41 \%$ \\
\hline URBANISMO & $14,85 \%$ & $47,22 \%$ & $46,46 \%$ & $5,17 \%$ & $28,21 \%$ \\
\hline GESTÃO AN & $9,30 \%$ & $33,77 \%$ & $43,69 \%$ & $0,00 \%$ & $22,00 \%$ \\
\hline ENERGIA & $7,65 \%$ & $29,86 \%$ & $10,30 \%$ & $0,00 \%$ & $12,47 \%$ \\
\hline ADMINISTRAÇÃO & $5,86 \%$ & $13,06 \%$ & $6,41 \%$ & $0,68 \%$ & $7,57 \%$ \\
\hline SEGURANÇA PÚBLICA & $7,71 \%$ & $18,96 \%$ & $8,69 \%$ & $0,00 \%$ & $6,95 \%$ \\
\hline TRANSPORTE & $6,35 \%$ & $1,82 \%$ & $7,52 \%$ & $1,21 \%$ & $3,86 \%$ \\
\hline ASSISTÊNCIA SOCIAL & $0,00 \%$ & $11,48 \%$ & $0,00 \%$ & $0,00 \%$ & $1,53 \%$ \\
\hline ENCARGOS ESPECIAIS & & $0,00 \%$ & $0,00 \%$ & & $0,00 \%$ \\
\hline cotal & $28,42 \%$ & $46,01 \%$ & $42,20 \%$ & $5,10 \%$ & $30,62^{\circ}$ \\
\hline
\end{tabular}

Fonte: Portal da Transparência do Distrito Federal - 2019.

Um aspecto relevante na avaliação da execução das emendas parlamentares indicadas é a capacidade executiva de cada órgão do Governo do Distrito Federal em concretizar a despesa autorizada em ações concretas, ou seja, que o recurso destinado seja empenhado e se torne uma entrega para população. É importante ressaltar que o um número expressivo em valores empenhados não significa uma eficiência na aplicação do recurso. Na Tabela 6, observamos que a função urbanismo tem o maior valor absoluto em despesas autorizadas e recursos empenhados entre 2016 e 2019, cerca de R\$ 654 milhões em despesa autorizada e R \$ 197 milhões em valores empenhados.

Porém, quando se realiza a proporção entre o valor empenhado por ação e despesa autorizada, verifica-se que o índice de empenho corresponde a pouco mais de $30 \%$ do valor inicialmente autorizado. A Novacap recebeu o maior volume de recursos em emendas parlamentares e executou pouco mais de $42 \%$ do valor inicial destinado (ver Tabela 7). 
Tabela 7 - Índice de Empenho, Despesa Autorizada e Empenho por Função e Órgão

\begin{tabular}{|c|c|c|c|}
\hline Função & $\%$ de empenho & Despesa Aut: & Empenho \\
\hline URBANISMO & $30,20 \%$ & RS654.183.625 & RS 197.576 .905 \\
\hline COMPANHIA URB. DA NOVA CAPITAL DO BRASIL-NOVACAP & $42.62 \%$ & $R \$ 322834.427$ & R5 137.597 .273 \\
\hline ADMINISTRAÇÃO REGIONAL DE CEILÂNDIA & $36,12 \%$ & $R \$ 44.709 .085$ & RS 16.150 .726 \\
\hline ADMINISTRAÇÃO REGIONAL DE BRAZLANDIA & $39,20 \%$ & $R \$ 23.794 .572$ & RS 9.326 .592 \\
\hline ADMINISTRAÇÃO REGIONAL DE ÁGUAS CLARAS & $12.97 \%$ & $R \$ 33.790 .000$ & $\operatorname{R5} 4.381 .249$ \\
\hline ADMINISTRAÇÃO REGIONAL DO RECANTO DAS EMAS & $23,12 \%$ & $R \$ 15.467 .856$ & $R .53 .575 .507$ \\
\hline SECRETARIA DE ESTADO DE OBRAS E INFRAEST. DO DF & $6,01 \%$ & $\mathrm{R} 555.847 .144$ & RS 3.356 .272 \\
\hline ADMINISTRAÇÃO REGIONAL DE SANTA MARIA & $23,50 \%$ & R512.750.000 & R5 2996.154 \\
\hline ADMINISTRAÇÃO REGIONAL DE SÃO SEBASTIÃO & $16.55 \%$ & R\$18.052000 & RS 2986.733 \\
\hline ADMINISTRAÇÃO REGIONAL DE PLANALTINA & $1441 \%$ & $R \$ 18200.000$ & $R 52622.114$ \\
\hline ADMINISTRAÇÃO REGIONAL DO PLANO PILOTO & $47,80 \%$ & RS4.140.000 & RS 1.978 .847 \\
\hline ADMINISTRAÇÃO REGIONAL DO ITAPOÂ & $22.12 \%$ & R\$7.800.000 & RS 1.725 .248 \\
\hline ADMINISTRAÇÃO REGIONAL DO PARANOÁ & $15,86 \%$ & $R \$ 10.030 .000$ & $\operatorname{RS} 1.590 .758$ \\
\hline ADMINISTRAÇÃO REGIONAL DO GAMA & $8.28 \%$ & R\$16.210.941 & RS 1.342 .036 \\
\hline COMPANHIA DE DESENV. DA HABITAÇÃO DO DF - CODHAB & $126,30 \%$ & RS1.000.000 & RS 1.262 .980 \\
\hline SECRETARIA DE AGRIC. ABASTECIMENTO E DESENV RURAL & $78,10 \%$ & RS1.500.000 & RS 1.171 .428 \\
\hline SERVIÇO DE LIMPEZA URBANA DO DISTRITO FEDERAL- SLU & $20,58 \%$ & R\$5.427.600 & RS 1.116 .905 \\
\hline ADMINISTRAÇÃO REGIONAL DE SOBRADINHO & $2485 \%$ & R\$3.650.000 & R5 907.076 \\
\hline ADMINISTRAÇÃO REGIONAL DA FERCAL - RA XOXI & $27,48 \%$ & R\$2300.000 & R5 631.943 \\
\hline ADMINISTRAÇÃO REGIONAL DO RIACHO FUNDO & $38,35 \%$ & RS1.600.000 & RS 613.535 \\
\hline ADMINISTRAÇÃO REGIONAL DE SAMAMBAIA & $409 \%$ & $R \$ 13.800 .000$ & R5 564.498 \\
\hline ADMINISTRAÇÃO REGIONAL DO RIACHO FUNDO II & $7,69 \%$ & RS6.600.000 & R5 507.624 \\
\hline TRANSPORTE URBANO DO DISTRITO FEDERAL - DFTRANS & $109,72 \%$ & $R S 400,000$ & $R \$ 438.894$ \\
\hline ADMINISTRAÇÃO REGIONAL SETOR COMPL. IND. E ABAST. & $13,40 \%$ & R\$3.130.000 & RS 419.507 \\
\hline ADMINISTRAÇÃO REGIONAL DE SOBRADINHO \| & $455 \%$ & R\$3.800.000 & R5 172.892 \\
\hline ADMINISTRAÇÃO REGIONAL DE TAGUATINGA & $0,51 \%$ & $R \$ 27350.000$ & RS 140.114 \\
\hline
\end{tabular}

Fonte: Portal da Transparência do Distrito Federal - 2019.

A função orçamentária cultura executada pelo órgão Secretaria de Cultura apresenta o maior índice de execução das despesas inicialmente alocadas, chegando ao índice de $88 \%$ de execução do recurso inicialmente aplicado. Em comparação, a função orçamentária educação executa no período pouco mais de $32 \%$ do valor destinado. (ver Tabela 8). 
Tabela 8 - Índice de Empenho, Despesa Autorizada e Empenho por Função e Órgão

\begin{tabular}{|c|c|c|c|}
\hline Função & $\%$ de empenho & Despesa Aut. & Empenho \\
\hline EDUCAÇÄO & $32,61 \%$ & R\$317.183.547 & RS 103.440 .991 \\
\hline SECRETARIA DE ESTADO DE EDUCAÇĀO DO DF & $3394 \%$ & RS298383.547 & RS 101.258 .406 \\
\hline ADMINISTRAÇÃO REGIONAL DE CELLÂNDIA & $13,79 \%$ & RS7.1500000 & R5 985.647 \\
\hline SECRETARIA DE ESTADO DE ESPORTE E LAZER DO DF & $41,67 \%$ & RS1200000 & RS 5000000 \\
\hline ADMINISTRAÇĀOO REGIONAL DE AGUAS CLARAS & & RSO & RS 396.938 \\
\hline COMPANHIA URB. DA NOVA CAPTALL DO BRASLL-NOVACAP & $287 \%$ & R510.450.000 & RS 3000000 \\
\hline CULTURA & $69,48 \%$ & RS87.705.856 & RS 60.937 .684 \\
\hline SECRETARIA DE EST. DE CULTURA DO DISTRTOO FEDERAL & $8824 \%$ & R547.462856 & Rs 41.880 .918 \\
\hline SECRETARIA DE EST. DE CULTURA E ECON. CRIAT. DO DF & $27,08 \%$ & R5288200000 & R5 7.804103 \\
\hline ADMINISTRAÇÃO REGIONALL DE BRAZLANDDAA & $12899 \%$ & RS2135000 & RS 2753.881 \\
\hline ADMINISTRAÇAOO REGONAL DO RECANTO DAS EMAS & $38477 \%$ & RS500.000 & RS 1.923 .850 \\
\hline ADMINISTRACCÃO REGONALL DE SÃOO SEBASTTÄO & $13758 \%$ & $R 5710000$ & R5 976.803 \\
\hline COMPANHIA URB. DA NOVA CAPTALL DO BRASIL-NOVACAP & $16635 \%$ & R5500000 & R5 $831.75:$ \\
\hline SEC DE POLITICAS P/CRIANÇAS, ADOLESC. EJUVENTUDE & $351,48 \%$ & $R S 200000$ & RS 702.954 \\
\hline ADMINISTRAÇAOO REGONAL DE SANTA MAR/A & & RSO & RS 667.339 \\
\hline SECRETARIA DE ESTADO DE ESPORTE E LAZER DO DF & $3594 \%$ & R\$1.700.000 & RS 610.967 \\
\hline ADMINISTRAÇAOO REGIONAL DE SOBRADINHO & $200,17 \%$ & $R \$ 300000$ & R\$ 600.521 \\
\hline ADMINISTRAÇĀO REGONAL SETOR COMPL IND. E ABAST. & $52243 \%$ & R580000 & RS 417.947 \\
\hline SECR. TRAB. DES. SOCIALL MULHERES IG. RACIALL DH & $3590 \%$ & R5780.000 & RS 280.000 \\
\hline ADMINISTRAÇÃO REG ONAL DE SOBRADINHO \| & $21881 \%$ & RS123.000 & RS 269.138 \\
\hline ADMINISTRAÇÃO REGONAL DO PARANOÁ & $11600 \%$ & RS180000 & RS 208.793 \\
\hline ADMINISTRAÇAOO REGONAL DO GAMA & $870 \%$ & R\$2350000 & RS 204.490 \\
\hline ADMINISTRAÇĀO REG ONAL DE SAMAMBAIA & $11933 \%$ & RS150:000 & RS 178.988 \\
\hline ADMINISTRAÇÃO REG ONAL DO RIACHO FUNDO & $29.94 \%$ & RS500000 & RS 149.689 \\
\hline SECRETARIA DE AGRIC. ABASTECIMENTO E DESENV RURAL & $133,66 \%$ & RS100000 & RS 133.660 \\
\hline ARQUIVO PÚBLCO DO DISTRTOO FEDERAL & $1569 \%$ & R\$815:000 & RS 127.879 \\
\hline ADMINISTRAÇÃO REGONAL DO GUARÁ & $37.83 \%$ & RS300000 & RS 113.488 \\
\hline ADMINISTRACC̆ÃO REGONAL DA FERCAL - RA XOXI & & RSO & RS 100.525 \\
\hline
\end{tabular}

Fonte: Portal da Transparência do Distrito Federal - 2019.

Um importante aspecto de análise é o nível de despesa autorizada para parlamentares considerados da base do governo em relação a parlamentares considerados como oposição ou independentes. No gráfico 6 é possível verificar que deputados considerados da base de governo entre legislaturas têm quase 2 vezes mais despesas autorizadas em relação aos parlamentares considerados como oposição ou independentes (ver Gráfico 6). Ressalta-se que todas as emendas alocadas são inicialmente classificadas como "bloqueadas" apenas com autorização do Poder Executivo elas podem ser executadas, passando para o status de despesa autorizada. 
Gráfico 6 - Percentual de Despesa Autorizada por Atuação e Ano

atuacao banCADA_BASE banCADA_BASE_oposicaO banCADA_oposICAO BASE INDEPENDENTE OPOSICAO
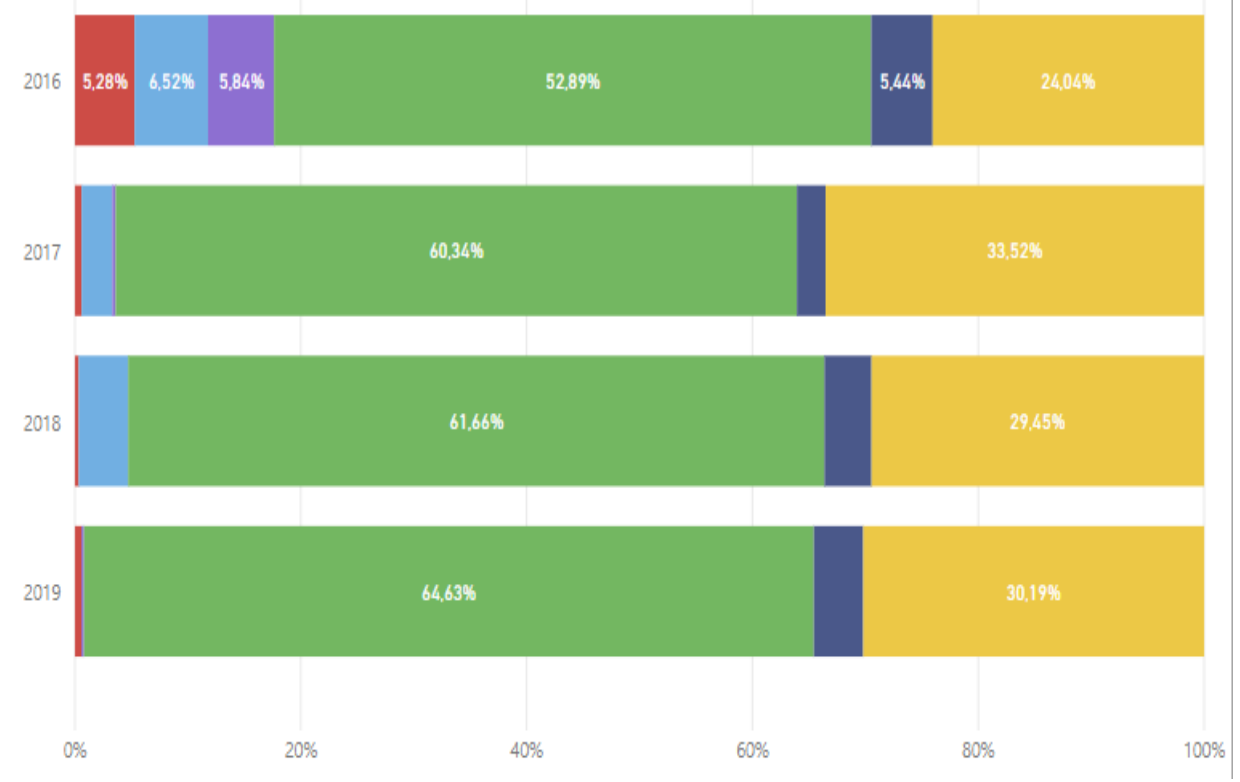

Fonte: Portal da Transparência do Distrito Federal - 2019.

Ao analisar o índice de execução de empenho em relação ao valor inicialmente destinado, observamos que parlamentares considerados independentes têm uma melhora performance na execução dos recursos inicialmente destinados (ver Gráfico 9). Em alguns momentos parlamentares se organizam para uma destinação em conjunto das emendas parlamentares. Esta estratégia é utilizada para aumentar o índice de execução da emenda parlamentar.

Tabela 9 - Percentual de Despesa Autorizada por Atuação e Ano

\begin{tabular}{l|rrrrr}
\multicolumn{2}{l}{2016} & 2017 & 2018 & 2019 & \multicolumn{1}{c}{ Total } \\
\hline INDEPENDENTE & $51,56 \%$ & $98,28 \%$ & $61,67 \%$ & $1,83 \%$ & $\mathbf{4 8 , 1 1} \%$ \\
BASE & $41,22 \%$ & $42,77 \%$ & $47,53 \%$ & $6,82 \%$ & $\mathbf{3 4 , 0 1 \%}$ \\
BANCADA_BASE_OPOSICAO & $8,68 \%$ & $51,30 \%$ & $42,58 \%$ & $\mathbf{2 8 , 5 3 \%}$ \\
OPOSICAO & $15,52 \%$ & $46,63 \%$ & $42,79 \%$ & $2,91 \%$ & $\mathbf{2 8 , 1 8 \%}$ \\
BANCADA_BASE & $3,58 \%$ & $274,24 \%$ & $0,00 \%$ & $0,00 \%$ & $\mathbf{2 6 , 7 8 \%}$ \\
BANCADA_OPOSICAO & $0,79 \%$ & $66,90 \%$ & & $0,00 \%$ & $\mathbf{3 , 8 5 \%}$ \\
Total & $\mathbf{2 9 , 1 4 \%}$ & $\mathbf{4 7 , 1 4 \%}$ & $\mathbf{4 6 , 3 5 \%}$ & $\mathbf{5 , 3 7 \%}$ & $\mathbf{3 2 , 1 1 \%}$
\end{tabular}

Fonte: Portal da Transparência do Distrito Federal - 2019.

Com o intuito de averiguar a relação entre as duas variáveis despesa autorizada e valor empenhado, foi aplicado o experimento com um modelo de regressão linear: "A regressão linear encontra a linha que melhor representa as variáveis de entrada com a variável de saída simples”, utilizando técnicas de machine learning. Para esta etapa foi utilizada a biblioteca sklearn para linguagem Python, com a importação do modelo linear_regression. 
A primeira etapa foi a verificação da correlação entre as duas variáveis pelo coeficiente de Pearson, a saber "[...] o coeficiente de correlação de Pearson, também chamado de 'coeficiente de correlação produto-momento' ou simplesmente de ' $\rho$ de Pearson' mede o grau da correlação (e a direção dessa correlação - se positiva ou negativa) entre duas variáveis de escala métrica".

Gráfico 7 - Correlação entre as variáveis Despesa Autorizada e Empenho

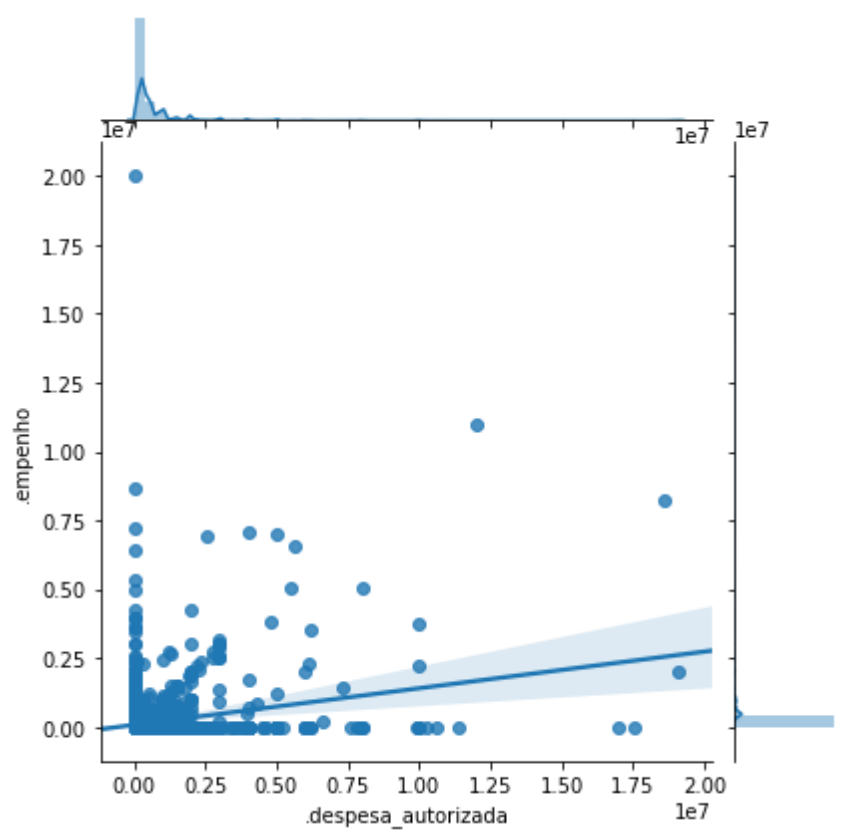

Fonte: Elaborado pelo autor.

O conjunto de dados foi divido entre $70 \%$ para treinamento do modelo e $30 \%$ para o conjunto de teste. Após as etapas de construção e validação do modelo observamos a conclusão do modelo de regressão linear, que para cada real aplicado em despesa autorizada retorna-se $\mathrm{R} \$ 0,38$ em valores empenhados (ver Gráfico 7).

\section{CONCLUSÃO}

O presente estudo buscou analisar a aplicação das emendas parlamentares realizadas por deputados do Distrito Federal, desse modo, evidenciou-se como é realizada a aplicação e execução das emendas propostas por parlamentares.

Concluímos que os parlamentares destinam recursos prioritariamente para obras e urbanismo, porém, mesmo com alto valores destinados, possuem baixa execução. Verificou-se que a função classificada como cultura é a melhor que executa recursos oriundos de emendas parlamentares. Foi possível constatar que o executivo executa pouco mais de $32 \%$ da despesa originalmente autorizada. Finalmente observou-se que 
parlamentares considerados como de base de governo têm duas vezes mais recursos autorizados quando comparados a parlamentares considerados como oposição ou independência. Em contrapartida, parlamentares considerados independentes têm um melhor índice de recursos de emendas parlamentares.

Desse modo, apresentamos características e atributos das emendas parlamentares destinada pelos deputados distritais do Distrito Federal entre os anos de 2016 e 2019. Foram considerados aspectos conceituais sobre emendas parlamentares no Distrito Federal. Questões como: índice de execução das emendas parlamentares, quais órgãos melhor executam emendas, quais funções orçamentárias recebem mais recursos e taxa de retorno em empenho das emendas foram apresentadas. Foi evidenciado que no período avaliado deputados considerados como base de governo tem um volume de liberação de recurso duas vezes maior que deputados considerados de oposição.

Assim o presente trabalho busca evidenciar aspectos da execução e efetividade do recurso destinado por parlamentares. 


\section{REFERÊNCIAS}

BATISTA, Mariana. A Conexão Ministerial: governo de coalizão e viés partidário na alocação de emendas parlamentares ao orçamento (2004-2010). Revista IberoAmericana de Estudos Legislativos, [S.1.], v. 4, n. 1, mai. 2015. Disponível em: <http://bibliotecadigital.fgv.br/ojs/index.php/riel/article/view/49202>. Acesso em: 12 fev. 2018

BRASIL. Constituição (1988). Constituição da República Federativa do Brasil. Brasília: Câmara dos Deputados, Coordenação de Publicações, 2015.

BRASIL. Lei Complementar n. 101, de 4 de maio de 2000. Estabelece normas de finanças públicas voltadas para a responsabilidade fiscal e dá outras providências. Diário Oficial [da] República Federativa do Brasil, Brasília, DF, 05 mai. 2000. Seção 1, p. 1.

BRASIL. Lei $n^{\mathbf{0}}$ 4.320, de 17 de março de 1964. Estatui normas gerais de Direito Financeiro para elaboração e controle dos orçamentos e balanços da União, dos Estados, dos Municípios e do Distrito Federal. Brasília, 1964. Disponível em:

<http://www.planalto.gov.br/ccivil_03/leis/14320.htm>. Acesso em: 26 jul. 2017.

BRASIL. Resolução n 1 de 2006 do Congresso Nacional. Dispõe sobre a Comissão Mista Permanente a que se refere o $\S 1^{\circ}$ do art. 166 da Constituição e sobre a tramitação das matérias a que se refere o mesmo artigo. Brasília, dez. 2006. Disponível em: <https://www2.camara.leg.br/legin/fed/rescon/2006/resolucao-1-22-dezembro-2006548706-normaatualizada-pl.html>. Acesso em: 26 jul. 2017.

DISTRITO FEDERAL. Lei Orgânica do Distrito Federal. Brasília-DF, 8 de junho de 1993. Disponível em: <https://www.cl.df.gov.br/pesquisa-de-leis-e-proposicoes>. Acesso em: 26 jul. 2017.

DISTRITO FEDERAL. Sistema de Gestão Governamental. Secretaria de Fazenda, Planejamento, Orçamento e Gestão do Distrito Federal, 2019. Disponível em: <http://cidadao.sisconep.df.gov.br/>. Acesso em: 26 jul. 2017.

DISTRITO FEDERAL. Programa de Descentralização Administrativa e Financeira (PDAF). Secretaria de Estado de Educação, Distrito Federal, 2019. Disponível em: $<$ http://www.se.df.gov.br/programa-de-descentralizacao-administrativa-e-financeirapdaf/>. Acesso em: 26 jul. 2017. 